\title{
Influencia de los márgenes de las restauraciones sobre la salud gingival
} The influence of margins of restorations on gingival health

\author{
Ardila Medina $\mathrm{CM}^{*}$
}

\section{RESUMEN}

Se ha evaluado durante un tiempo la relación entre las restauraciones dentales y su condición periodontal. Las investigaciones han demostrado que la colocación de márgenes subgingivales juega un papel importante debido a que proveen un nicho ecológico para los periodontopatógenos. La localización del margen gingival de una restauración está directamente relacionada con el estado de salud de los tejidos periodontales y existen dos factores que pueden influir desfavorablemente: el grado de adaptación marginal y la profundidad intracrevicular de la línea de terminación de la preparación. Aún cuando el control de estas variables fundamentales no es uniforme en la mayoría de estudios, se pueden considerar algunas conclusiones importantes a partir de la evidencia científica, con el fin de que los clínicos evalúen cómo los tejidos periodontales responden a las restauraciones protésicas.

Palabras clave: Restauración dental, restauración subgingival, salud gingival.

\section{SUMMARY}

The relationship between dental restorations and periodontal status has been examined for some time. Research has shown that subgingival margin placement play an important role in providing an ecologic niche for periodontal pathogens. The location of gingival margin of a restoration is directly related to the health status of the adjacent periodontium and there are two factors that could adversely influence the periodontal response, the degree of marginal adaptation and the intracrevicular depth of the preparation finish line. Although control of important variables was not uniform among the studies, important conclusions can be garnered from this cientific evidence that elevates one's awareness of how periodontal tissues respond to prosthodontic restorations.

Key words: Dental restoration, subgingival restoration, gingival health.

Fecha de recepción: 9 de enero de 2009.

Aceptado para publicación: 14 de enero de 2009.

* Profesor Asistente Universidad de Antioquía. Miembro Junta Directiva Asociación Colombiana de Periodoncia y Oseointegración.

Ardila Medina CM. Influencia de los márgenes de las restauraciones sobre la salud gingival. Av. Odontoestomatol 2010; 26 (2): 107-114.

\section{INTRODUCCIÓN}

El conocimiento de la respuesta de los tejidos periodontales a las restauraciones con coronas artificiales y a las prótesis fijas (PF) es fundamental cuando se realizan planes de tratamiento con pronósticos predecibles. Durante el diagnóstico y plan de tratamiento es necesario considerar la evidencia científica disponible. La investigación clínica se ha enfocado sobre el efecto de las restauraciones indirectas sobre los 
tejidos periodontales aunque también existen algunas publicaciones que consideran la asociación entre restauraciones directas y salud gingival. Diferentes estudios han demostrado que la pobre adaptación marginal (1), la localización de márgenes gingivales intracreviculares profundos (2), las superficies rugosas de la restauración (3) y las restauraciones sobrecontorneadas (4) pueden contribuir a una inflamación periodontal localizada. La inflamación se presenta debido a que estas restauraciones pueden proveer un ambiente protegido en el cual la microflora normal madura hacia una periodontopatógena $(5,6)$. El objetivo de este artículo es presentar las investigaciones que realiza la literatura científica considerando la respuesta de los tejidos periodontales a las restauraciones, coronas individuales y PF, haciendo énfasis en aspectos como la inflamación, pérdida de inserción, profundidad de sondaje y pérdida ósea.

\section{INFLAMACIÓN}

La gran mayoría de estudios concluyen que la coronas y PF contribuyen a la inflamación periodontal. Valderhaug y colaboradores (2), realizaron un estudio longitudinal que se extendió por un periodo de 15 años. Se llevó a cabo en un grupo de 102 pacientes que recibieron $108 \mathrm{PF}$. El índice gingival (IG) (7), fue superior en los dientes restaurados con coronas observándose principalmente en aquellas que presentaron márgenes subgingivales.

831 pacientes atendidos regularmente participaron en un estudio con el fin de evaluar el índice de placa (IP) (8) y la inflamación gingival en sujetos que recibían atención odontológica regular (9). Se observó mayor inflamación gingival en aquellos dientes restaurados con coronas y PF que presentaban márgenes subgingivales, aún en aquellos pacientes que recibían cuidado dental preventivo frecuentemente.

Con el fin de evaluar la relación entre la adaptación marginal de restauraciones coladas y el estado de salud periodontal, se seleccionaron 44 restauraciones con márgenes subgingivales que llevaran en boca por lo menos cuatro años en 29 sujetos escogidos aleatoriamente (1). Se encontró una fuerte correlación entre la discrepancia marginal e IG aumentado
(7) y entre discrepancia marginal e incremento del volumen de fluido crevicular.

Peumans y colaboradores (10), evaluaron la influencia de restauraciones con resina sobre los tejidos periodontales marginales. Las restauraciones fueron colocadas directamente sobre 79 dientes anteriores superiores en 19 sujetos. Todos fueron llamados cinco o seis años después para una valoración periodontal. Se evaluaron el IP (8), el IG (7) y la profundidad de sondaje en los sitios bucal proximal de todos los dientes restaurados unilateralmente. Se realizó una comparación intraindividual de la salud periodontal entre los dientes restaurados y los dientes intactos. El IP $(p=0,029)(8)$ y el IG $(p=0,008)(7)$ fueron significativamente más altos cuando se compararon con los dientes intactos. Por otra parte, a diferencia en la profundidad de sondaje estuvo cercana al nivel de significancia $(p=0,059)$. De esta manera los autores concluyeron que las resinas directas que permanecen en boca por más de cinco años tienen una influencia negativa sobre la salud periodontal marginal, la cual incluye aumento en la retención de placa, inflamación gingival y destrucción periodontal.

Se realizó una investigación en 103 pacientes (de los cuales 39 eran fumadores) con el fin de examinar la encía marginal de 255 dientes restaurados: 101 resinas, 98 amalgamas y 56 incrustaciones de oro (11). Las restauraciones seleccionadas deberían llevar en boca más de seis meses. La encía marginal en contacto con los materiales restauradores se evaluó mediante el IG (8), el índice de sangrado (IS) (12), la profundidad de sondaje y el grado de movilidad dental. Como controles se emplearon 255 dientes contra laterales sin restauraciones. Se observó ausencia de sangrado al sondaje en el 25,7\%, 55, $1 \%$ y 58,9\% de los dientes restaurados con resina, amalgama e incrustaciones de oro respectivamente. Todos los dientes restaurados presentaron diferencias significativas al compararse con los dientes control. Se encontró una diferencia significativa entre fumadores y no fumadores en el grupo restaurado con resinas. El IS $(p=0,0005)(12)$ y el IG $(p=0,0106)$ (8) fueron significativamente superiores en el grupo de fumadores en comparación a los no fumadores. Los investigadores concluyeron que la adaptación marginal de los dientes restaurados examinados presen- 
tó diferencias específicas en sus materiales. La manipulación de resinas en comparación a la amalgama y el oro fue muy sensible y en consecuencia prevalecía un error en la técnica. La alta prevalencia de inflamación gingival asociada con las resinas fue causada por aplicaciones no indicadas, fallos en la técnica o simplemente debida a las propiedades químicas de los materiales.

Diez odontólogos generales revisaron 240 pacientes con 480 coronas metal cerámicas en un ensayo clínico prospectivo realizado con el fin de cuantificar el impacto de los márgenes coronales sobre la salud gingival de dientes posteriores (13). Se analizó el efecto de la colocación de un margen coronal sobre el sangrado del surco gingival y la acumulación de placa bacteriana mediante modelos de regresión. La posibilidad de sangrado en las coronas con márgenes intrasulculares fue aproximadamente el doble que el de aquellas con márgenes supragingivales. Los sitios bucales, comparados con los linguales, mostraron una probabilidad menor de sangrado sulcular y el tipo de aleación no influyó sobre la salud gingival.

Schätzle y colaboradores (14), realizaron una investigación con el propósito de evaluar la relación a largo plazo entre las restauraciones dentales y la salud periodontal. Sus datos derivaron de un estudio longitudinal a 26 años realizado en hombres escandinavos de clase media, caracterizados por higiene oral entre moderada y buena, además de asistencia a revisiones odontológicas regulares. Se revisaron las superficies bucales y mesiales en cada uno de los siete exámenes realizados entre 1969 y 1995 . El grupo experimental estaba constituido por 98 restauraciones subgingivales y se empleó un grupo control con 615 restauraciones localizadas $1 \mathrm{~mm}$ por encima del margen gingival. Los autores concluyeron que en todos los puntos examinados durante 26 años, el grado de inflamación de la encía adyacente a restauraciones subgingivales fue muy superior comparado con aquellos dientes sin restauraciones o con los que presentaron restauraciones $1 \mathrm{~mm}$ por encima del margen gingival.

En la figura 1 se observa la inflamación gingival ocasionada por una corona en porcelana con margen subgingival.

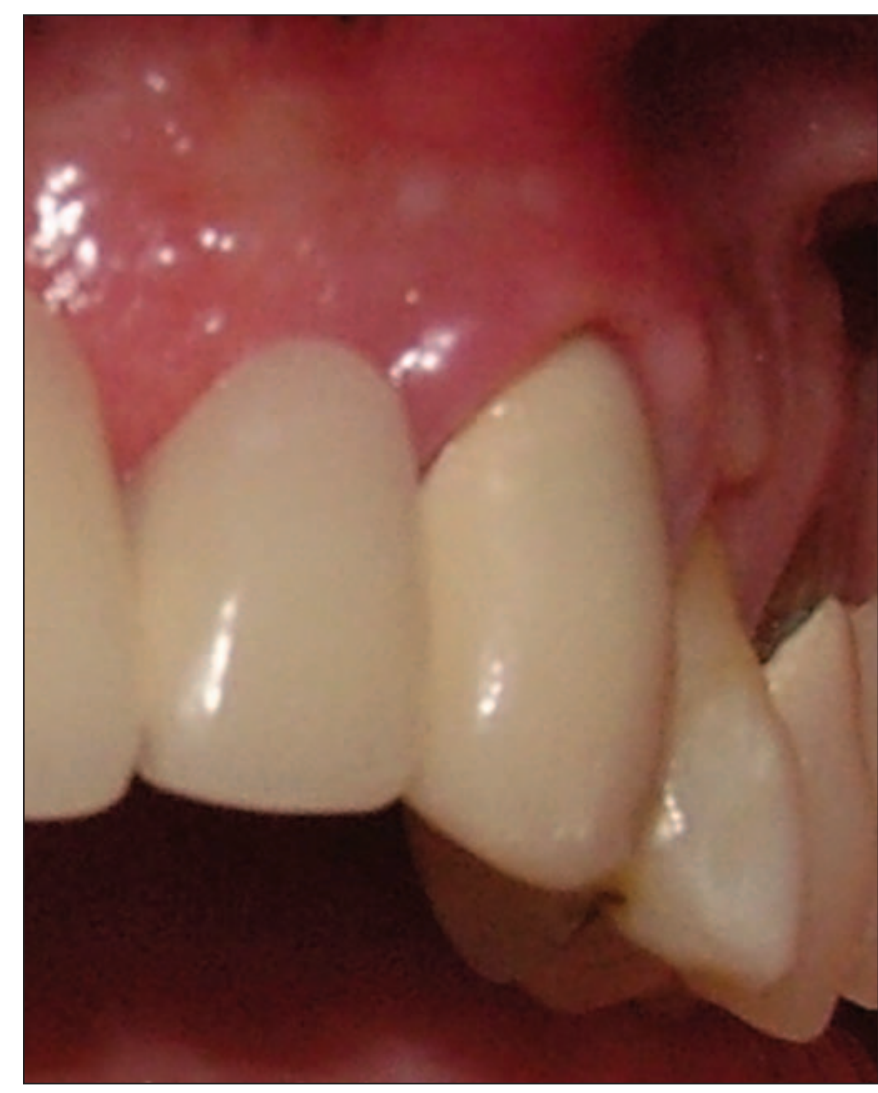

Fig. 1. Inflamación gingival asociada a corona con margen subgingival.

\section{PÉRDIDA DE INSERCIÓN (PI)}

Gran cantidad de investigaciones han presentado la asociación entre las restauraciones subgingivales y la PI. El estudio de Schätzle y colaboradores (14), concluye como en las valoraciones realizadas al tercer y cuarto año después de la evaluación inicial se observó una diferencia significativa en el promedio de PI entre los grupos experimental y control. Los primeros mostraron una PI de 0,78 $\mathrm{mm}$ y en los controles fue de 0,57 mm. Después de 19 años el promedio acumulado de la PI aumentó a 1,58 mm para el grupo experimental y 1,12 para el grupo control, diferencias que fueron estadísticamente significativas. A partir de esta investigación se deduce que la localización subgingival de las restauraciones produce una notable PI la cual puede ser detectada uno a tres años después de realizados los procedimientos restauradores.

Otro estudio comparó evaluaciones periodontales de dientes pilares y no pilares en 55 sujetos 15 años 
después de la colocación de PF (2). Los autores hallaron PI en los primeros cinco años posteriores a la cementación de la restauración.

Broadbent y colaboradores (15), estudiaron en una cohorte de individuos sí las superficies proximales restauradas actuaban como factor de riesgo para la PI. Se examinaron 884 superficies dentales en 914 sujetos cuando tenían 26 y 32 años de edad. Los investigadores concluyeron que en aquellos sitios donde se había realizado una restauración antes de los 26 años, la PI a los 32 en el mismo sitio, tenía dos veces más probabilidad de ser $=$ a $3 \mathrm{~mm}$ que si la superficie del diente permanecía sin restaurar. Esta asociación persistía aún después de controlar por potenciales confundidores como el hábito de fumar.

Se realizó una investigación con el fin de determinar la influencia de las dimensiones gingivales sobre la PI y la aparición de recesiones (16). La población de estudio estaba constituida por 11 sujetos periodontalmente saludables a quienes se les había instalado 44 coronas en porcelana y 39 dientes sirvieron como controles. Se realizaron exámenes periodontales inmediatamente después de la instalación de las coronas y a los 3, 6, 9 y 12 meses posteriores. Doce meses después, los dientes restaurados con coronas experimentaron un promedio en la PI de 0,17 $\pm 0,99$ $\mathrm{mm}$ y en promedio el margen gingival había migrado apicalmente $0,43 \pm 0,74 \mathrm{~mm}$. En el análisis multivariado, considerando la estructura correlacionada de los datos, identificó las coronas instaladas como el principal factor causal de la PI, especialmente en dientes que presentaban surcos gingivales poco profundos y bandas estrechas de encía insertada. La figura 2 muestra la migración apical del margen gingival en algunos pilares posterior a la cementación de una prótesis fija.

Se realizó un estudio clínico prospectivo a dos años para determinar si la colocación de los márgenes proximales de las coronas dentro de la zona de amplitud biológica ocasionaba PI (17). En 41 pacientes, se examinaron 116 dientes preparados y 82 dientes contra laterales sin restaurar sanos periodontalmente. Se realizaron evaluaciones a los 3, 6, 12 y 24 meses después de la preparación dental y se midió la distancia entre el margen de la restauración y la cresta alveolar. Los dientes con restauraciones fue-

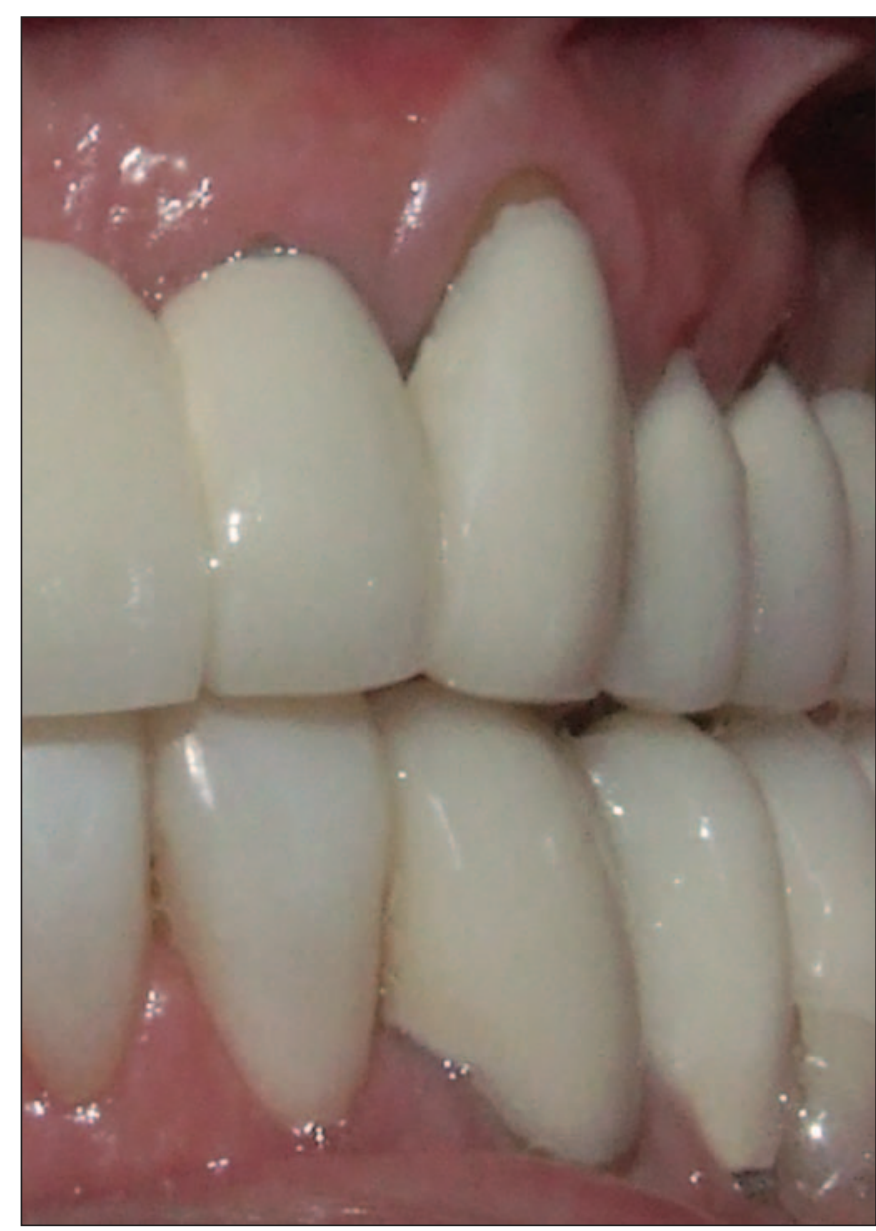

Fig. 2. Recesión gingival posterior a cementación de prótesis fija.

ron divididos en tres grupos según la distancia existente entre el margen de la corona y la cresta alveolar: grupo 1, menor a $1 \mathrm{~mm}$ (los márgenes de la restauraciones se ubicaron en el tejido conectivo supraalveolar); grupo 2, entre 1 y 2 mm (los márgenes de la restauraciones se ubicaron en el epitelio de unión); grupo 3, mas de 2 mm (los márgenes de la restauraciones se ubicaron intracrevicularmente) Adicionalmente, se midió la amplitud vestibular de la encía queratinizada. Los investigadores encontraron alteraciones significativas en el nivel de inserción en los grupos 1 y 2 . En el grupo 1 se presentó una PI promedio de 0,6 $\pm 0,1 \mathrm{~mm}$ y en el grupo 2 la PI fue de 0,2 $\pm 0,1 \mathrm{~mm}$, mientras que en el tercer grupo la PI fue de 0,2 $\pm 0,1 \mathrm{~mm}$. En el grupo control no se encontraron cambios significativos relacionados con la PI. Por otra parte, la dimensión de la encía queratinizada disminuyó de 5,3 a 5,1 mm en el grupo 1 . 
Pihlstrom (18), estableció que en estudios de seguimiento acerca de la salud periodontal, el objetivo de interés principal debe ser determinar radiográficamente el nivel de inserción, agrega sin embargo, que la profundidad de sondaje también debe ser registrada.

\section{PROFUNDIDAD DE SONDAJE (PS).}

Diferentes estudios en los cuales los sujetos fueron seguidos hasta por 15 años después de la inserción de coronas, PF y restauraciones registran PS entre 0.05 y 0.5 mayores que los controles $(2,9,11,13$, $14,19-23)$.

Jansson y colaboradores (24), investigaron la influencia de la placa bacteriana sobre la profundidad de la bolsa periodontal adyacentes a restauraciones proximales en amalgama después de tratamientos periodontales no quirúrgicos. A partir de 120 pacientes seleccionados aleatoriamente se incluyeron 200 superficies de molares o premolares con restauraciones subgingivales (grupo experimental) y 200 sitios contra laterales sin restaurar en el mismo paciente (grupo control). A nivel base la PS promedio en el grupo experimental fue significativamente más profunda $(0,25 \mathrm{~mm})$ que en los controles. Después del tratamiento y al examinarse nuevamente la PS promedio adyacente a las restauraciones proximales con placa se encontró que eran significativamente mayores que sus controles $(0,34 \mathrm{~mm})$. Los autores concluyeron que las restauraciones subgingivales con sus márgenes localizados subgingivalmente aún después del tratamiento periodontal, deben ser consideradas un factor de riesgo para el progreso de la periodontitis.

Willershausen y colaboradores (11), evaluaron la influencia de diferentes tipos de restauraciones en contacto directo con los tejidos gingivales. Todos los dientes restaurados presentaron diferencias significativas en la PS cuando se compararon con los dientes control. La profundidad de sondaje promedio para los dientes restaurados con resina fue de 3,6 mm (control 2,4 mm), para los dientes con amalgama fue de $3 \mathrm{~mm}$ (control $2.4 \mathrm{~mm}$ ) y para los restaurados con incrustaciones de oro fue de $2,5 \mathrm{~mm}$ (control 2,1).
Bader y colaboradores (9), evaluaron 831 pacientes atendidos regularmente en clínicas odontológicas de Carolina del Norte. Análisis específicos por superficie mostraron una PS significativamente mayor en aquellas restauraciones con márgenes subgingivales $(\mathrm{p}<0,05)$.

El estudio longitudinal realizado por Valderhaug y colaboradores (2) encontró que en 343 dientes pilares restaurados se incrementó levemente la PS, mientras que en 525 dientes control permaneció en el mismo nivel durante los 15 años de seguimiento que abarcó la investigación.

\section{PÉRDIDA ÓSEA (PO)}

La PO puede ser determinada mediante interpretación visual, medidas directas o análisis de imagen digital en radiografías. Se ha considerado que la medida más precisa es el uso de radiografías estandarizadas que permiten medir cambios pequeños de hasta $0,2 \mathrm{~mm}$, cuando se emplean procedimientos computarizados (25). En la figura 3 se observa pérdida ósea relacionada con márgenes subgingivales de una restauración

El principal objetivo del estudio realizado por Kovács y colaboradores (26), fue evaluar como la irritación local y la retención de placa causada por coronas con márgenes subgingivales afectaba la pérdida ósea en pacientes con periodontitis crónica. La incidencia de factores que favorecían la retención de placa bacteriana fue evaluada en 200 radiografías panorámicas aleatoriamente seleccionadas del archivo del departamento de Periodoncia de una clínica de Budapest. Radiográficamente, el 82,5\% de las restauraciones presentaron adaptación marginal inadecuada. La PO promedio fue de 5,4 mm con tendencia a ser mayor a medida que incrementaba la edad y en presencia de restauraciones. 113 sujetos presentaron una PO mayor a $4 \mathrm{~mm}$.

Keglevich y colaboradores (27), realizaron un estudio clínico con el fin de evaluar el papel de las restauraciones subgingivales en el avance de la periodontitis en un grupo de pacientes aleatoriamente seleccionados. El nivel de PO alrededor de dientes con restauraciones fue comparado con el de dientes 


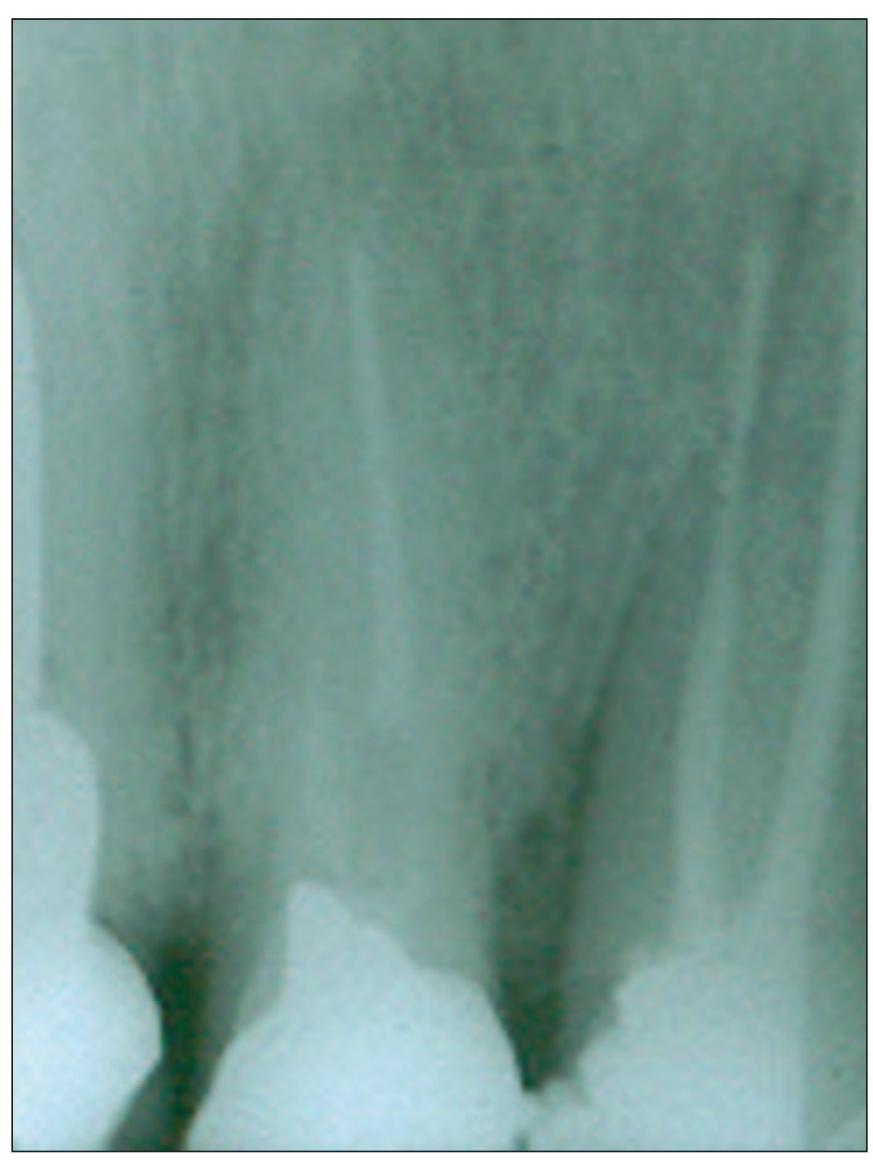

Fig. 3. Pérdida ósea relacionada con restauración subgingival.

contra laterales en 170 radiografías panorámicas. La PO fue mayor en los dientes restaurados, diferencias que fueron estadísticamente significativas $(p<0,01)$.

Fueron seleccionados 101 sujetos con el fin de examinar el efecto de las restauraciones dentales, coronas y obturaciones sobre los tejidos periodontales (28). Los resultados mostraron mayor gingivitis, bolsas periodontales y pérdida ósea en los dientes con restauraciones comparado con sus respectivos controles contra laterales.

Albandar y colaboradores (29), evaluaron la relación entre restauraciones dentales subgingivales y el avance de la enfermedad periodontal en 227 adolescentes en un periodo de tres años. Los sujetos fueron examinados clínica y radiográficamente al inicio y una vez al año. Se encontró una asociación significativa entre las restauraciones defectuosas y pérdida de soporte periodontal.
Se realizó una investigación en 423 márgenes coronales que fueron divididos en 355 subgingivales y 68 supragingivales (30). Los tejidos gingivales tendían a sangrar 2,42 veces más frecuentemente con márgenes subgingivales y a su vez tenían 2,65 más posibilidades de presentar recesiones gingivales y PO cuando se comparaban con sus controles contra laterales.

La tabla 1 resume los estudios que respaldan la respuesta de los tejidos periodontales a los márgenes de las restauraciones.

\section{CONCLUSIONES}

Las restauraciones con coronas y prótesis fijas incrementan la incidencia y el progreso de la inflamación gingival. Los estudios han demostrado que en promedio el índice gingival (7) es mayor en los dientes restaurados que en los controles contra laterales. Algunos factores relacionados con la restauración como la colocación de márgenes intracreviculares, deficiente adaptación marginal, contornos inadecuados y superficies rugosas se asocian con inflamación.

Aun cuando algunos estudios han informado aumento en la profundidad de sondaje después de la cementación de coronas y prótesis fijas, sus resultados deben ser evaluados individualmente dependiendo del diseño de investigación.

La evidencia no ha demostrado que las restauraciones ocasionen una rápida pérdida ósea, por ello, es

\section{TABLA 1.- RESPUESTA DE LOS TEJIDOS PERIODONTALES A LOS MÁRGENES DE LAS RESTAURACIONES}

\begin{tabular}{|ll|}
\hline Inflamación & $(1,2)(9-11)(13,14)$ \\
\hline Pérdida de inserción & $(2)(14-18)$ \\
\hline $\begin{array}{l}\text { Aumento en la profundidad } \\
\text { del sondaje }\end{array}$ & $\begin{array}{l}(2)(9)(11)(13,14) \\
(19-24)\end{array}$ \\
\hline Pérdida ósea & $(26-30)$ \\
\hline
\end{tabular}


muy importante evaluar críticamente la literatura y la metodología de las investigaciones publicadas con el fin de obtener información concluyente. Sin embargo, es imperativa la realización de restauraciones que cumplan con criterios protésico periodontales adecuados.

\section{BIBLIOGRAFÍA}

1. Felton DA, Kanoy BE, Bayne SC, Wirthman GP. Effect of in vivo crown margin discrepancias on periodontal health. J Prosthet Dent 1991;65:357-64.

2. Valderhaug J, Ellingsen JE, Jokstad A. Oral hygiene, periodontal conditions and carious lesions in patients treated with dental bridges. A 15-year clinical and radiographic follow-up study. J Clin Periodontol 1993;20:482-9.

3. Bollen CM, Papaioanno W, Van Eldere J, Schepers E, Quirynen M, van Steenberghe D. The influence of abutment surface roughness on plaque accumulation and peri-implant mucositis. Clin Oral Implants Res 1996;7:201-11.

4. Gómez F. Ardila CM. Contornos y perfil de emergencia: aplicación clínica e importancia en la terapia restauradora. Av Odontoestomatol 2009; 25:171-9.

5. Parkinson CF. Excessive crown contours facilitate endemic plaque niches. J Prosthet Dent 1976; 35:424-9.

6. Yotnuengnit B, Yotnuengnit P, Laohapand P, Athipanyakom S. Emergence angles in natural anterior teeth: influence on periodontal status. Quintessence Int 2008; 39:126-33

7. Silness J, Löe H. Periodontal disease in pregnancy 3. Response to local treatment. Acta Odontol Scand 1966;24:747-59.

8. Löe H, Silness J. Periodontal disease in pregnancy1. Acta Odontol Scand 1963;21: 53351.

9. Bader JD, Rozier RG, McFall WT Jr, Ramsey DL. Effect of crown margins on periodontal conditions in regularly attending patients. J Prosthet Dent 1991;65:75-9.

10. Peumans $M$, Van Meerbeek B, Lambrechts $P$, Vanherle G, Quirynen M. The influence of direct composite additions for the correction of tooth form and/or position on periodontal health. A retrospective study. J Periodontol 1998;69:422-7.

11. Willershausen B, Köttgen C, Ernst CP. The influence of restorative materials on marginal gingiva. Eur J Med Res 2001;6:433-9.

12. Edwards RC. Bleeding index. A new indicator in personal plaque control. J Am Soc Prevent Dentistry 1975;5:20-2.

13. Reitemeier B, Hänsel K, Walter MH, Kastner C, Toutenburg $\mathrm{H}$. Effect of posterior crown margin placement on gingival health. J Prosthet Dent 2002;87:167-72.

14. Schätzle M, Land NP, Anerud A, Boysen H, Bürgin W, Löe $H$. The influence of margins of restorations of the periodontal tissues over 26 years. J Clin Periodontol 2001;28:57-64.

15. Broadbent JM, Williams KB, Thomson WM, Williams SM. Dental restorations: a risk factor for periodontal attachment loss? J Clin Periodontol 2006;33:803-10.

16. Koke U, Sander C, Heinecke A, Müller HP. A possible influence of gingival dimensions on attachment loss and gingival recession following placement of artificial crowns. Int J Periodontics Restorative Dent 2003;23:439-45.

17. Günay H, Seeger A, Tschernitschek H, Geurtsen W. Placement of the preparation line and periodontal health -a prospective 2-year clinical study. Int J Periodontics Restorative Dent 2000;20:171-81.

18. Pihlstrom B. Issues in the evaluation of clinical trials of periodontitis: a clinical perspective. J Periodontal Res 1992;27:433-41.

19. Valderhaug J. Periodontal conditions and carious lesions following the insertion of fixed prostheses: 
a 10-year follow-up study. Int Dent J 1980;30: 296-304.

20. Muller HP. The effect of artificial crown margins at the gingival margin on the periodontal conditions in a group of periodontally supervised patients treated with fixed bridges. J Clin Periodontol 1986; 13:97-102.

21. Orkin DA, Reddy J, Bradshaw D. The relationship of the position of crown margins to gingival health. J Prosthet Dent 1987;57:421-4.

22. Koth DL. Full crown restorations and gingival inflammation in a controlled population. J Prosthet Dent 1982;48:681-5.

23. Konradsson K, van Dijken JW. Effect of a novel ceramic filling material on plaque formation and marginal gingiva. Acta Odontol Scand 2002;60: $370-4$.

24. Jansson L, Blomster S, Forsgårdh A, Bergman $\mathrm{E}$, Berglund $\mathrm{E}$, Foss $\mathrm{L}$ et al. Interactory effect between marginal plaque and subgingival proximal restorations on periodontal pocket depth. Swed Dent J 1997;21:77-83.

25. Benn DK. Limitations of the digital image subtraction technique in assessing alveolar bone crest changes due to misalignment errors during image capture. Dentomaxillofac Radiol 1990;19: 97-104.
26. Kovács V, Tihanyi D, Gera I. The incidence of local plaque retentive factors in chronic periodontitis. Fogorv Sz 200;100:295-300.

27. Keglevich T, Ratkóczi L, Dóri F, Gera I. Effect of the quality of restorations and of dental prosthesis on the alveolar bone loss in patients with chronic destructive periodontitis. Fogorv Sz 2000;93: 225-32.

28. Tiwarri OS, Salimeno T Jr, Choksi S, Rao MS, Gupta OP. Effects of restorations and carious lesions on the periodontium in humans. Ann Dent 1992;51:22-5.

29. Albandar JM, Buischi YA, Axelsson P. Caries lesions and dental restorations as predisposing factors in the progression of periodontal diseases in adolescents. A 3-year longitudinal study. J Periodontol 1995 ; 66:249-54.

30. Orkin DA, Reddy J, Bradshaw D. J The relationship of the position of crown margins to gingival health. J Prosthet Dent 1987; 57: 421-4.

\section{CORRESPONDENCIA}

Carlos Martín Ardila Medina

Carrera 47 No. 20 sur 46 Envigado

Antioquia-Colombia

57(4) 3348122

E-mail: cmartin@odontologia.udea.edu.co 